\title{
Adolescences Hypertension: A Late Case of Coarctation of Aorta: A Case Report and Short Review of Literature
}

\author{
Andreas C Petropoulos ${ }^{1,2,3}$, Medine Haciyeva ${ }^{2}$, Necile Rzazade ${ }^{2}$, Aynur Xudiyeva $^{1}$ and \\ Ibrahim Isayev ${ }^{2}$ \\ ${ }^{1}$ Merkezi Klinika, Baku, Azerbaijan \\ ${ }^{2}$ Azerbaijan Medical University Hospital, Azerbaijan \\ ${ }^{3}$ The "Aziz Aliyev" post graduate and CME national medical training center, Baku-Azerbaijan
}

\begin{abstract}
Received: 08 January, 2018; Accepted: 02 February, 2018; Published: 05 February, 2018
*Corresponding author: Dr. Andreas C Petropoulos, Department of Pediatrics (Cardiology), Azerbaijan Medical University, Educational and Therapeutically Hospital, Azerbaijan, E-mail: andrepetropoulos@gmail.com
\end{abstract}

\begin{abstract}
Coarctation of the Aorta $(\mathrm{CoA})$ is a common Congenital Heart Disease (CHD) presenting with many symptoms and signs, in any age group. From its dramatic appearance as a part of Hypoplastic Left Heart Syndrome(HLHS), to early Congestive Heart Failure(CHF)in neonatal and early infantile age, to undetected Hypertension (HTN) in adolescence or even late adulthood. The aim of this case report and short review is to present this unique CHD, underline the presentation of late detected CoA, the use of cardiac CT to assess severity and plan surgical treatment. Finally, to discuss the bourdon of treating resistant HTN even after successful surgical treatment. This difficult to control by pharmacotherapy, HTN and the late complications of persistent uncontrolled HTN for the Cardiovascular System(CVS), are the principle reasons for these patients to have a long-life follow-up. This population, while aging, will face important and increasing morbidity and mortality.
\end{abstract}

Keywords: Coarctation of Aorta; Late Detection of CoA; Hypertension Secondary to CoA; Cardiac CT Scan

Abbreviations: CoA:arctation of the Aorta; re-CoA: Recoarctation; CHD: Congenital Heart Disease; HLHS: Hypoplastic Left Heart Syndrome; HTN: Hypertension; DA: Ductus Arteriosus; IAA: Interrupted Aortic Arch; BP: Blood Pressure; LCOS: Low Cardiac Output Syndrome; CHF: Congestive Heart Failure; DAo: Descending Aorta; (pm)VSD: perimembranus Ventricular Septal Defect; BAov: Aortic Bicuspid valve; AovS: Aortic valve stenosis; PDA: Patent Ductus Arteriosus; Mv: Mitral valve; LVOT: Left Ventricular Outflow Tract; ECG: Electrocardiography;Echo-2D: Transthoracic Echocardiography2D; LVPO or VO: Left Ventricular Pressure Overload or Volume Overload; VSD: Ventricular Septal Defect; c-MRI: cardiac Magnetic Resonance Imaging; cv-CT: cardiovascular Computer Tomography; CAD: Coronary Artery Disease; CVS: Cardiovascular System; BA: Balloon Angioplasty; LVH: Left Ventricular Hypertrophy; LV: Left Ventricular; b-Blockers: beta-Blockers; ACE-I: ACE inhibitors; ARBs: Angiotensin Receptor Blockers

\section{Introduction}

Although CoA has been thought to be a narrowing of the lumen of the isthmus, adjacent to the Ductus Arteriosus (DA) on the posterior wall of the thoracic aorta is mostly then that a serious type of angiopathy of the Aorta [1]. CoA is a common Congenital Heart Defect (CHD) found in approximately 1 case per 2500 live births and is the fifth most common type of CHD, with a favorable prevalent in males [2]. This frequency underestimates delayed diagnosis, even in the adult population, due to many late presenting cases [3]. CoA is an aortopathy that lies clinical, within a spectrum of arch abnormalities ranging from a discrete narrowing, pre-and-post DA, to a long segment of arch hypoplasia, even reaching an Interrupted Aortic Arch (IAA). These two entities, is believed today to have different mechanisms [3]. It can also involve early histologic changes that can create intracranial aneurysms suggests that wall abnormalities are not confined only to the aorta [4]. Clinical, can present in any age from Fetal life as a part of the Hypoplastic Left Heart

Syndrome(HLHT), to early neonatal life, approximately at the end of the first week of life when the DA closes and the narrowness of the CoA diminishes the flow of the oxygenated blood to the periphery of the body. This presents, as a Low Cardiac Output Syndrome (LCOS) with Congestive Heart Failure (CHF), with increasingly worsening acidosis, acute renal failure and finally early death, that mimics late neonatal sepsis $[1,5]$. If we are faced with a case of a more favorable anatomy with a mild and short narrowing of the lumen area, of the arch or the Descending Aorta (DAo) this can present in any age from childhood to adulthood either during an investigation of a murmur or due to incidental diagnosis of HTN. Frequently we can find the combination of a complex not - cyanotic CHD that involves a perimembranus ventricular septal defect [(pm) VSD] with a Bicuspid Aortic valve (BAov) that can be clinical normal or have any amount of stenosis (AovS) or regurgitation [1,5]. CoA is present in $18 \%$ of patients with Turner syndrome [6]. In Williams's syndrome, a congenital 
and multisystem genetic disorder, mostly associated with supravalvular aortic stenosis and peripheral pulmonary artery branches stenosis. CoA, are present in $10 \%$ of patients with William's syndrome $[7,8]$. Finally, CoA can also be presenting congenital cardiovascular anomalies involving multiple left-sided lesions, including Shone's complex and HLHS $[1,5,7,8]$. The less frequent but more tragic presentation can be a rapture of intracranial aneurisms. These can be found in $5 \%$ of patients suffering from CoA. Now-a-days, MRI scans report a prevalence of intracranial aneurysms in patients with CoA approximately of $10 \%[8,9]$. This is five times more common than the average population. In one study, HTN was more common in the population of CoA patients with intracranial aneurysms [9]. The diagnosis is based on the combination of medical history, physical examination and imaging techniques [5]. As mention above, clinical presentation of CoA differs significantly by age. CHF in neonatal life or chest pain, exercise intolerance (due to LCOS on exercise) or due to claudication (secondary to lower extremity ischemia) has been reported in older children [8]. Investigation of patients with accidental findings of "new" systolic or continues murmurs (collateral vessels around the CoA), mostly in the intracapular area, must alert the physician. Most important, is the detection of HTN with a reverse phenomenon in which the BP in the upper is higher than in the lower extremities, by at least $20 \mathrm{mmHg}$. This finding with additional diminished or delayed femoral arterial pulses has been thought in the past to be the gold standard findings that points directly to CoA [5]. Newly studies show that these clinical findings are only present in near $60 \%$ of patients suffering from CoA [10]. Additional evidence can be added by a Chest X-Ray, showing a "figure of three" sign formed by the aortic nob, the stenotic segment, and the dilated post-stenotic segment of the aorta, suggesting CoA (Figure 1). The heart silhouette can
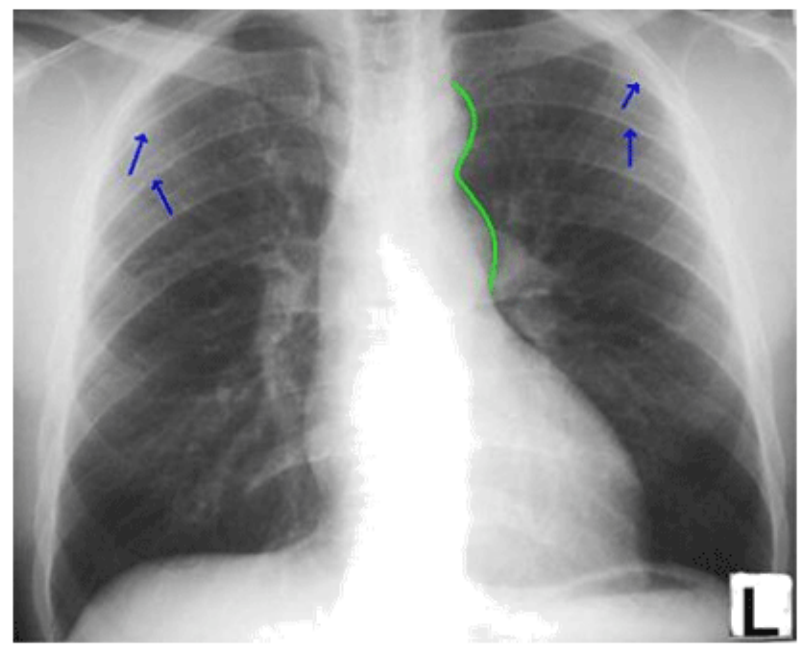

Figure 1: Chest X-Ray in CoA (See the green line describing the "figure 3 sign" formed by the aortic nob, the stenotic segment, and the dilated post-stenotic segment of the aorta suggesting CoA. The heart silhouette can be normal or mildly enlarged. The blue arrows point to existing inferior rib notching can also be seen in the third to eighth ribs bilaterally caused by the presence of dilated intercostal collateral arteries) be normal or mildly enlarged. Inferior rib notching can also be seen in the third to eighth ribs bilaterally caused by the presence of dilated intercostal collateral arteries. Electrocardiography (ECG) can demonstrate in late onset patients unexplained evidence of Left Ventricular Hypertrophy (LVH), from chronic Left Ventricular Pressure Overload(LVPO)[5] (Figure 2).

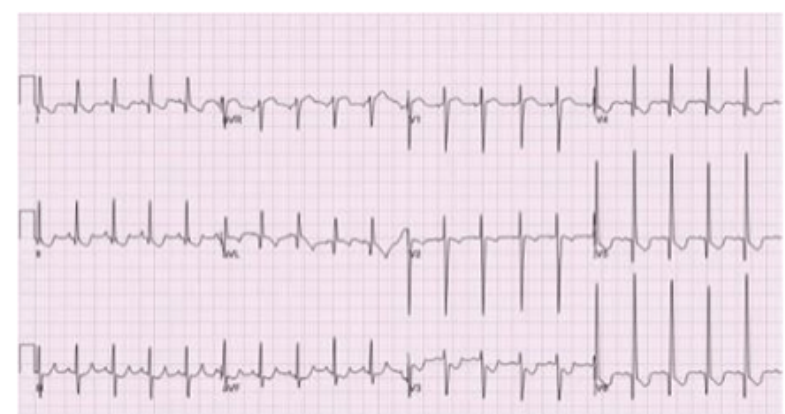

Figure 2: ECG in CoA. (See the (L) axis deviation of the QRS and LVH due to the LVPO. Notice ischemic changes in T- waves (negative T- waves), due to strain of the LV secondary to chronic sever HTN)

A variety of diagnostic methods can be added to prove the existence of CoA and quantify its severity. Less invasive, cheap and easy to provide in all levels of care, today is Echocardiography (Echo-2D) [10]. As CoA is "classically" divided to pre- and - post DA, we will follow this classification when describing Echo-2D and Computer Tomography (CT) angiography findings. In the pre-ductal type of CoA, the inner diameters of distal aortic arch and aortic isthmus narrow significantly, and the aortic arch and/ or DAo reveal an irregular stricture. In the post ductal CoA type, the DAo constriction is located distal to the left subclavian artery presenting a gourd-shaped appearance [10] (Figure 3). In postductal type, the distal DAo presents a dilatation after the CoA area

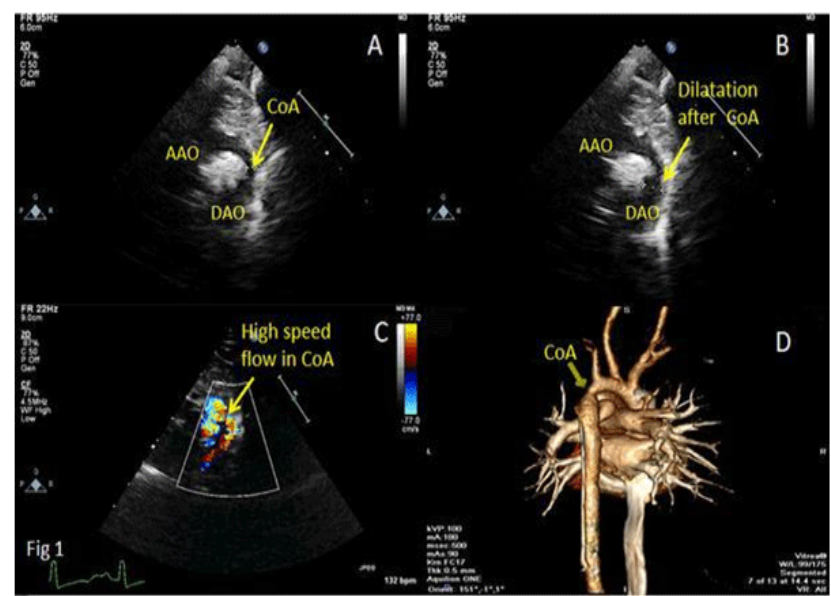

Figure 3: TTEcho-2D and CT) (Transthoracic echocardiograms in suprasternal view of a 2-month-old boy showing[A] the location of the coarctation of aorta $(\mathrm{CoA})$ between the AAo and DAo, $[\mathrm{B}]$ dilatation of the aorta after the CoA, and [C] high speed flow in the CoA. Computed tomographic angiography [D] obtained at 5 months of age confirmed the location of the CoA [10] 
[10] (Figure 4). Color mapping and Doppler examination improves the diagnostic accuracy [5,10]. Meanwhile Echo-2D will detect any additional anatomic lesions as discussed above as well as the function of the CVS $[5,8,10]$.

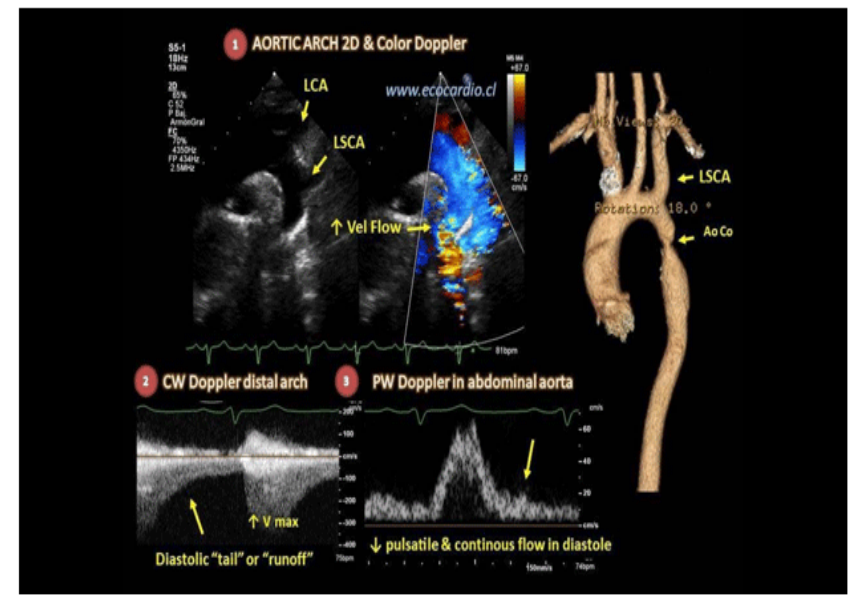

Figure 4: TTEcho-2D and CT (Transthoracic echocardiograms in suprasternal view of a 2-month-old boy showing [1] the location of the CoA between the distal to DAo with dilatation of the aorta before the CoA, [2] and [3] high speed flow in DAo due to CoA and continues flow in Abdominal Aorta. Computed tomographic angiography [4] obtained in the same age patient confirming the $\mathrm{CoA}[10])$

Cardiac catheterization, once, the golden standard for diagnosis of CoA , today has a limited diagnostic role but remains essential in the interventional management of patients with $\operatorname{CoA}[8,10]$. New imaging techniques as cardiac Magnetic Resonance Imaging (c-MRI) and cardiovascular Computer Tomography (cv-CT) are frequently used. Gadolinium-enhanced c-MRI angiography and 3D reconstruction images, provides excellent resolution of cardiac anatomy and vascular structures. Using phase contrast flow analysis, we can estimate the peak gradient across the CoA area [11]. Although the benefits of c-MRI are huge, this imaging technique is still expensive and limited to centers of excellence in treating CHD. On the other hand, the use of cV- CT must be considered in selected patients, as those who carry pacemakers or implantable cardioverter defibrillators that are not c-MRI compatible [12]. Other advantages of cv-CT include, improved image resolution, shorter scan time, and greater availability across different institutions. CT angiography is also used to assess concomitant coronary anomalies that may not be so well visualized with c-MRI. Considerable variations in measurements between the two techniques have been reported in the same patient, highlighting the importance of using one specific modality in serial assessment [13]. The users of cv-CT assessing repaired CoA, adhering to radiation safety principles, must minimize radiation dose, as a regulatory requirement [6]. Today, regarding age groups and previous or none used repair (native CoA), patients can be offered both interventional and/or surgical options of treatment $[16,14]$. In patients with a native CoA or re-CoA, gradient greater or equal to $20 \mathrm{~mm} \mathrm{Hg}$, is an indication for treatment. Patients with longstanding native $\mathrm{CoA}$ with significant collateral flow, have a lower measured gradient despite severe CoA. Therefore, patients with extensive collaterals should undergo repair, even in lower gradients. With many different options, deciding on the optimal treatment strategy for CoA can be complicated, as there is no comprehensive evidence-based standard of care or algorithm [14]. The 2008, Guidelines from the American College of Cardiology and the American Heart Association provide some insight, but the level of evidence supporting these recommendations are suboptimal [15]. In principle, management is based on the age of presentation, complexity of the anatomy, and whether the CoA represents a native vs recurrent obstruction. For infants or young children presenting with native CoA, most centers prefer surgical repair due to the long-term risk of aneurysm following BA, the need for re-dilatation with stent placement in near future, and the limitations imposed by small arteries unable to accommodate larger sheath sizes [16]. However, BA can be considered as a palliative strategy in stabilizing critical ill neonates considered too sick for immediate surgical repair [8]. Surgical repair may also, be more appropriate in patients with complex CoA anatomy, including those with transverse arch obstruction, tortuous segments of re-CoA, distortion of adjacent arterial branches, or when repair of associated cardiac defects is required $[8,15]$. In the older child (weight over $25 \mathrm{Kg}$ ), adolescent, or adult presenting with a simple, small segment, juxtaductal, native CoA, stent placement is considered a reasonable approach, offering a less invasive alternative to surgical intervention and good long term outcomes [6]. Only stents expandable to an adult size should be used, to avoid later surgical intervention [16]. For recurrent $\mathrm{CoA}$ in the younger child, it is reasonable to consider initial BA, as aneurysm is less of a long-term concern than with native CoA BA is variably successful, and surgical reintervention may be required when there is incomplete relief of obstruction $[17,8]$. Stent placement can also be considered for recoarctation in older children and adolescents when the stent can be dilated to near adult size, avoiding the need for multiple redilations [17].

\section{Case Report}

The patient we present is a 14year, Azerbaijani male (date of Birth 17/04/2003) that was admitted for long standing pain in his legs after exercise and periodical headaches. The complains started gradually from the age of four years old firstly the leg pains and then from the age of seven years old the headaches. Specifically, the leg pains were noticed during playing outdoors and mostly after the first ten minutes of intense activities. Pain got worse as exercise was continuing. Finally, was relieved a few minutes after resting with no need of further medication. After the age of seven years old the patient started to complain about headaches. They were observed less than once a month, mostly in the afternoon hours of the day, with duration of one to two hours. The use of ordinary nonsteroid anti-inflammatory medication abolished the pain.

His Family history outlined a maternal history of tension headaches during her adolescence and paternal essential HTN, firstly detected at age 40 years old. The family stated that the child was regularly followed up by a pediatrician.

The Physical Examination reviled a well-developed male, with both height, weight and head circumference measures between the 50th and 75th centile. He was pink well perfused and without 
respiratory distress. A soft continuance murmur in the left subscapular area and the left sub-clavicle area of II/VI was noticed. The pulses on the upper extremities were bounding when compared with the lower extremities. The femoral pulses were weak. The BP measurement on the right hand revealed a BP of 150/60 mmHg. Lower extremity BP was measured at 100/50 mm Hg.

His Chest X-Ray, showed elements of CoA. The Echo-2D revealed a normal basic anatomy with a normal atrioventricular and ventriculoarterial connections. The Pulse Doppler trace of the descending aorta demonstrated a continues flow during the cardiac cycle (Figure 5). A trivial tricuspid valve regurgitation Doppler measurement estimated an elevated systolic pressure in the RV of $50 \mathrm{mmHg}$. The LV was globally hypertrophic. A bicuspid Aortic valve was present with a trivial Aov regurgitation. Overall the cardiac function was normal. From the suprasternal notch view a

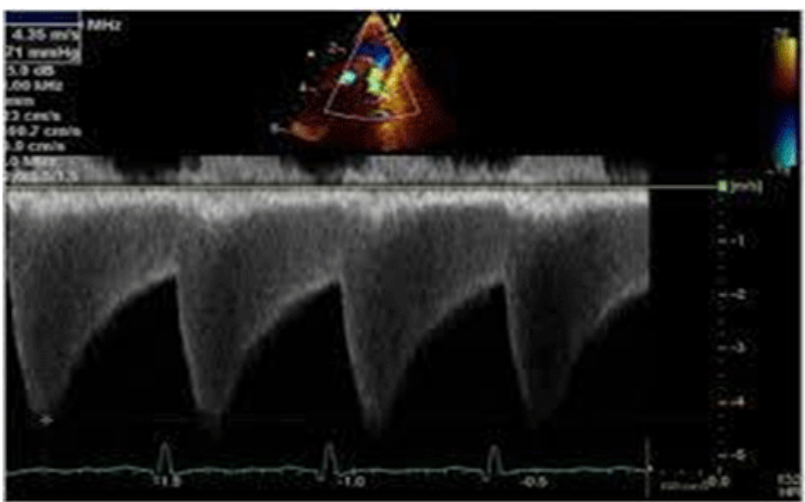

Figure 5: TTEcho-2D (Showing the post ductal CoA of our patient and the Gradient and Diastolic continues flow of our patient)
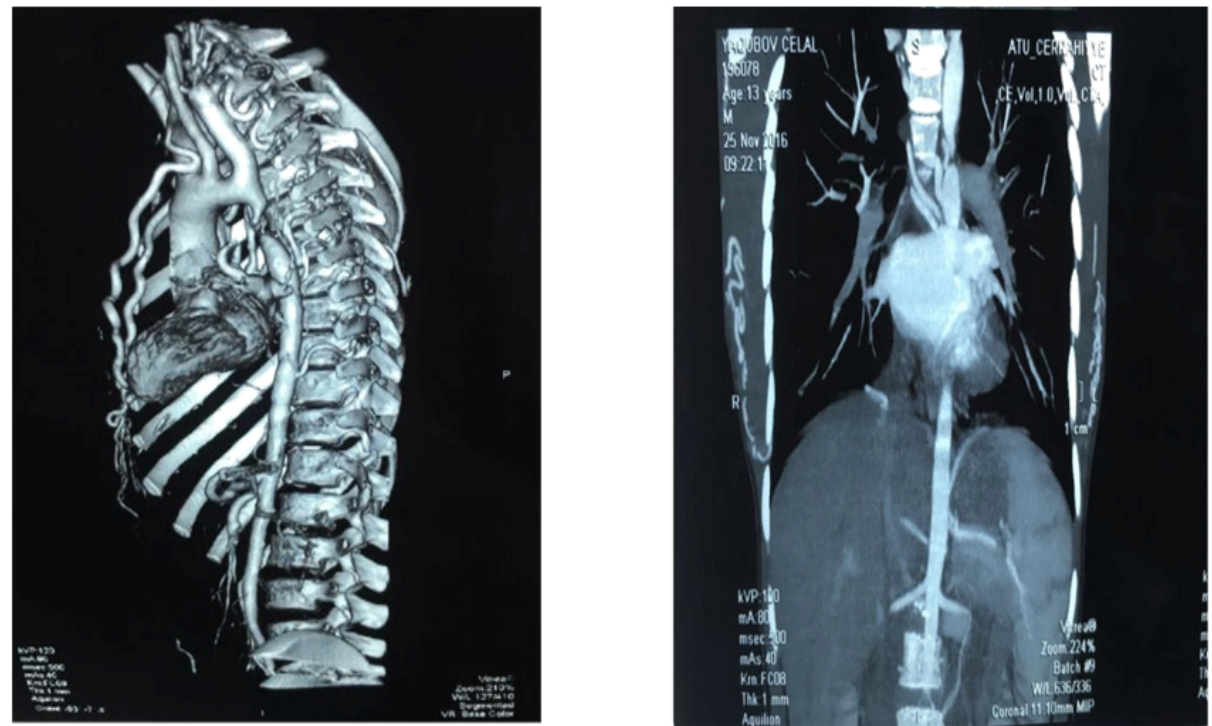

Figure 6-7: cv-CT (of the patient showing the coarcted post-ductal long and extremely narrowed area and the collateral vessels that preserve the continuity in flow to the distal DAo)

moderate dilated AAo a huge left subclavian artery and a narrowing of the DAo two centimeters distal to the isthmus was noticed. The gradient across the stenosis was max 58/mean $36 \mathrm{mmHg}$ with a doppler signal of a continuity in diastole (diastolic tail) (Figure 6).

With these findings, it was obvious that the patient had secondary to CoA, HTN stage- 1 and that the coarcted area was not only post ductal located but was also possible a long in length and extremely narrowed in size area, possible bypassed by collateral vessels.

For these reasons we selected as our next imaging technique, $\mathrm{cv}$-CT as a classical angiography that cannot lead to interventional treatment would be unnecessary in this case, due to the suspected anatomy. The $\mathrm{cv}$-CT revealed the full anatomy of the defect. Also showed the existence of collateral vessels permitting continuity in blood supply to the distal DAo (Figure 6,7).
Patient and his family was consultant for the findings of the investigation, the final diagnosis and the plan of treatment. Based on our findings we commenced the patient on ACE-i (Enalapril 5 mg BD) and referred to cardiovascular surgical repair in June 2017. Waiting for repair and on a maximum dose of Enalapril(10mg BD) we added Amlodipine (a calcium channel blocker) $5 \mathrm{mg}$ BD to control the HTN of the patient.

As this was a native, late presenting CoA with a long segment and collateral vessels, in a patient with a BAov and minimal regurgitation, we suggested surgical repair.

He was successfully operated in late June 2017, using a prosthetic patch of Polytetrafluoroethylene (PTFE) across the stenotic area, without resection of the coarctation ridge, by an off pump, left thoracotomy, and a fourth intercostal incision. The collateral vessels were preserved. The cross - clamping time was 24 minutes (Figure 8). During the post- operative 84 hours stay in 


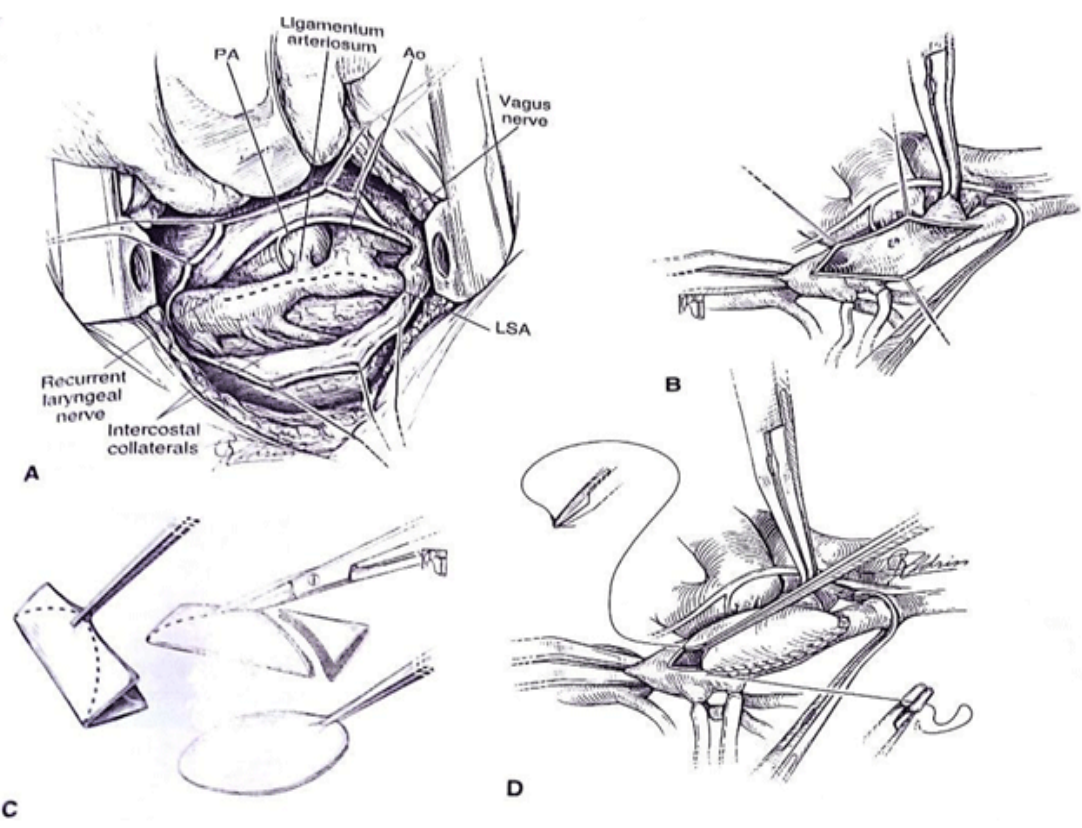

Figure 8:Showing the Patch (PTFE) Aortoplasty, adopted by Pediatric Cardiac Surgery [18]

cardiac intensive care unit i he presented with both early and late paradoxic post- operative HTN that was tolerated by a combination of Milrinone (phosphodiesterase 3 inhibitor) that reduces LV afterload and increases contractility and a combination of ACE-i (enalapril) and b-blocker(Esmolol) given iv. He was discharged from the hospital after seven days on treatment with Enalapril 10 mg BD and Amlodipine $5 \mathrm{mg}$ BD.

He was followed up on a month, three months and six months appointments postoperatively. Although the Echo-2D did not review any gradient across the previous CoA area and the ambulatory BP 24 hours Holter gave us normal mean BP's measurements an exercise test revealed exercise induced HTN reaching at the fourth stage of a Bruce protocol a maximum BP of $190 / 70 \mathrm{mmHg}$ that reduced to $150 / 60 \mathrm{mmHg}$ at the first minute of recovery. Further evaluation of "organ-targets" of HTN by, fundoscopy, GFR measurement, urine analysis for microalbuminuria and LV mass index, showed evidence of longstanding HTN. Taking also in consideration the early paternal history of essential HTN, these findings, increase the possibility of our patient to have a live long HTN with all the negative consequences it carries. For this reason, we will follow closely this young man.

\section{Discussion}

CoA is a very common disease, accounting for $6-8 \%$ of live births with CHD [8]. Despite the early presentation in neonatal life, many cases can be mist- as the one presented - if BP is not obtained in each well baby visit after the age of three years old as the American Academy of Pediatrics guidelines suggest [19].In our case, the two main symptoms of the patient were leg pains and headaches. Initially, leg pains were noticed after the first ten minutes of intense activities and got worse as exercise was continuing, at age 4 years old. Pain was relieved a few minutes after resting automatically. As he grows the leg pains became somehow less intensive. So, what was lost on this patient was claudication that was thought by others to be growing pains. Regarding the headaches. In medicine an anecdotal relationship between headaches and HTN exists. Recent studies have shown that Mild (140 to $159 / 90$ to $99 \mathrm{mmHg}$ ) or moderate (160 to179/100 to $109 \mathrm{mmHg}$ ) chronic arterial HTN does not appear to cause headache. Ambulatory blood pressure monitoring in patients with mild and moderate HTN has shown non-convincing relationship between blood pressure fluctuations over a 24-hour period and presence or absence of headache [2021].So, these headaches were mostly tension type. The element of the family history with a young father suffering from HTN and the physical examination findings needed to be considered and by measuring the BP as early as three years old would have accelerated the diagnosis of our patient.

The use of non-invasive imaging techniques as cv-CT gave us accurint diagnosis and permitted the best plan of further surgical treatment. Recognizing the benefits of c-MRI and cv-CT the 2008 American College of Cardiology/American Heart Association Guidelines for the Management of Adults with Congenital Heart Disease recommend that patients with CoA to be initially diagnosed and followed- up at least every 5 years [22].

Regarding the strategy of treatment followed in this patient. A late presenter -over $25 \mathrm{~kg}$ body weight-and even more if he/ she are adolescence, in many centers around the world, could be a candidate for an interventional treatment. As this was a native, CoA with a long segment and collateral vessels, in a patient with a BAov and minimal regurgitation, we suggested surgical repair. This was 
also the suggestion of our Interventional Cardiologist, mostly based on native CoA the long coarctation segment. Surgical repair was our unanimous decision.

The disappointed element of the case was the post- operative persisting CoA. Further, the evaluation of "organ-targets" of HTN by fundoscopy, renal evaluation and LV mass index, calculated by Echo-2D, showed evidence of longstanding HTN. In longer-term anti-HTN therapy is required, due to chronic HTN, following even successful treatment of the CoA with no residual gradient, anti-HTN medicines must be used aggressively to maintain normal BP. In 2008 the American College of Cardiology/American Heart Association Guidelines for the Management of Adults with CHD recommend use of an ACE-i, as first-line therapy. Additional treatment with b-blockers, Calcium channel blockers and Angiotensin receptor blockers can be used. When monotherapy is not successful -most of the cases of late CoA treatment-dual or even three classes of antiHTN medicines must be used to normalize BP [23].

As HTN can be seen early or late after even a successful treatment a lifelong prevention strategy is needed. Studies have shown that $30 \%$ of ex-CoA treated patients will suffer from HTN by adolescence raising to about $60 \%$ in adulthood. This suggests that HTN may be an inevitable consequence of CoA, even when an effective anatomical repair has been achieved early in life. For this reason, late detected patients will suffer from chronic HTN. Despite the progress in pharmacotherapy the best medicine treatment is debatable and will include more than one class of anti-HTN drugs. Primary prevention to these Patients will be as soon as possible to detect and early operate before the age of one's year old $[8,23,24]$. Secondary prevention would aim to eradicate any residual stenosis and treat existing HTN if present with an ACE-i or if not controlled on monotherapy to add other anti-HTN classes [25,26].Finally, tertiary prevention would be treating aggressively, lifelong HTN [24-26]. Further research into the mechanisms leading to HTN may identify therapies to target the vasculopathy changes seen in CoA and improve outcome [6,23-26].

\section{Conclusions}

CoA was thought to be, a discrete LH obstructive lesion, easy to detect and treat. Today, it is not only the fifth most common CHD but can also be easily mist, despite the progress in imaging techniques, even up to adulthood. Treatment strategies involve surgical and interventional procedures that have proven to have excellent early, mid and late term (up to 30 years), results. The most important morbidity is HTN. This can be seen as early as the lesion is detected or late after even 30 years of a successful treatment. Early surgery may prevent or delay the onset of HTN but in the end, most of the patients will present with HTN. These patients are at risk of premature CAD, LV systolic and diastolic dysfunction, and rupture of aortic or cerebral aneurysms as CoA is more that a regional stenosis. It is a vasculopathy. Long term follow-up by a multidisciplinary team of physicians in the field of pediatrics, internal medicine, nephrology, neurology and CHD is our best up to date, strategy.

\section{References}

1. Rudolf AM. Congenital Diseases of the Heart-Clinical \& Physiological Considerations. $3^{\text {rd }}$ ed. Wiley-Blackwell; 2009.

2. van der Linde D, Konings EE, Slager MA, Witsenburg M, Helbing WA, Takkenberg JJ, et al. Birth prevalence of congenital heart disease worldwide: a systematic review and meta-analysis. J Am Coll Cardiol. 2011;58(21):2241-2247. doi: 10.1016/j.jacc.2011.08.025

3. Strafford MA, Griffiths SP, Gersony WM. Coarctation of the aorta: A study in delayed detection. Pediatrics. 1982;69(2):159-163.

4. Connolly HM, Huston J, Brown RD Jr, Warnes CA, Ammash NM, Tajik AJ. Intracranial aneurysms in patients with coarctation of the aorta: a prospective magnetic resonance angiographic study of 100 patients. Mayo Clin Proc. 2003;78(12):1491-1499.

5. Allen HD, Gutgesell HP, Clark EB, Driscoll DJ. "Moss and Adams", Heart Disease in Infants, Children and Adolescents, including the Fetus and Young Adult. $6^{\text {th }}$ ed. Lippincott Williams \& Wilkins; 2001.

6. Nguyen L, Cook S. Coarctation of the Aorta. Strategies of improving outcome. Cardiol Clin. 2015;33(4):521-530, vii. doi: 10.1016/j. ccl.2015.07.011

7. McBride KL, Pignatelli R, Lewin M, Ho T, Fernbach S, Menesses A, et al. Inheritance Analysis of Congenital Left Ventricular Outflow Tract Obstruction Malformations: Segregation, Multiplex Relative Risk, and Heritability. Am J Med Genet A. 2005;134A(2):180-186.

8. Petropoulos AC, Moschovi M, Xudiyeva A, Qarayev F, Isayev I. Late Coarctation Presenters Suffer Chronic Hypertension Resisting to Medicine Treatment. Peertechz J Pediatr Ther. 2017;3(1):001-008.

9. Curtis SL, Bradley M, Wilde P, Aw J, Chakrabarti S, Hamilton M, et al. Results of Screening for Intracranial Aneurysms in Patients with Coarctation of the Aorta. AJNR Am J Neuroradiol. 2012;33(6):11821186. doi: 10.3174/ajnr.A2915

10.Sun Z, Cheng TO, Li L, Zhang L, Wang X, Dong N, et al. Diagnostic Value of Transthoracic Echocardiography in Patients with Coarctation of Aorta: The Chinese Experience in 53 Patients Studied between 2008 and 2012 in One Major Medical Center. PLoS One. 2015;10(6):e0127399. doi: 10.1371/journal.pone.0127399

11. Shepherd B, Abbas A, McParland P, Fitzsimmons S, Shambrook J, Peebles C, et al. MRI in adult patients with aortic coarctation: diagnosis and followup. Clin Radiol. 2015;70(4):433-445. doi: 10.1016/j.crad.2014.12.005

12. Rosenthal E, Bell A. Optimal coarctation stenting imaging after. Heart. 2009;96(15):1169-1171

13. Alfred Hager, Harald Kaemmerer, John Hess. Comparison of Helical CT Scanning and MRI in the Follow-up of Adults with Coarctation of the Aorta. Chest. 2015;127(6):2296.

14.Torok RD, Campbell MJ, Fleming GA, Hill KD. Coarctation of the aorta: Management from infancy to Adulthood. World J Cardiol. 2015;7(11):765-775. doi: 10.4330/wjc.v7.i11.765

15.Warnes CA, Williams RG, Bashore TM, Child JS, Connolly HM, Dearani JA, et al. Guidelines for the Management of Adults with Congenital Heart Disease: a report of the American College of Cardiology/American Heart Association Task Force on Practice Guidelines. J Am Coll Cardiol. 2008;52(23):e143-263. doi: 10.1016/j.jacc.2008.10.001

16. Cowley CG, Orsmond GS, Feola P, McQuillan L, Shaddy RE. Long term, randomized comparison of balloon angioplasty and surgery for native coarctation of the aorta in childhood. Circulation. 2005;111(25):34533456 . 
17. Feltes TF, Bacha E, Beekman RH 3rd, Cheatham JP, Feinstein JA, Gomes $\mathrm{AS}$, et al. Indications for cardiac catheterization and intervention in pediatric cardiac disease: a scientific statement from the American Heart Association. Circulation. 2011;123(22):2607-2652. doi: 10.1161/ CIR.0b013e31821b1f10

18. Mavroudis M, Backer CL. Pediatric Cardiac Surgery. Third Edition. Mosby; 2003

19. Expert Panel on Integrated Guidelines for Cardiovascular Health and risk reduction in Children and Adolescents: Summary report. Pediatrics. 2011;128(5).

20. Assarzadegan F, Asadollahi M, Hesami O, Aryani O, Mansouri B, Beladi M. Secondary headaches attributed to arterial hypertension. Iran J Neurol. 2013;12(3):106-110.

21. Vaughan CJ, Delanty N. Hypertensive emergencies. Lancet. 2000;356(9227):411-417.
22.Warnes CA, Williams RG, Bashore TM, Child JS, Connolly HM, Dearani JA, et al. (2008) ACC/AHA 2008 guidelines for the management of adults with congenital heart disease: a report of the American College of Cardiology/American Heart Association Task Force on Practice Guidelines. J Am Coll Cardiol. 2008;52(23):e143-263. doi: 10.1016/j. jacc.2008.10.001

23. Petropoulos A. Hypertension in the Young An overgrowing concern in Pediatrics 2end BITS, Annual World Conference in Pediatrics, Abstract Book. Tayhan: China; 2014.

24. Giordano U, Cifra B, Giannico S, Turchetta A, Calzolari A. Mid-term results, and therapeutic management, for patients suffering hypertension after surgical repair of aortic coarctation. Cardiol Young. 2009;19(5):451-455. doi: $10.1017 /$ S1047951109990734

25. Chu PY, Campbell MJ, Miller SG, Hill KD. Anti-hypertensive drugs in children and adolescents. World J Cardiol. 2014;6(5):234-244. doi: 10.4330/wjc.v6.i5.234

26. Flynn JT. Management of hypertension in the young role of antihypertensive medications. J Cardiovasc Pharmacol. 2011;58(2):111120. doi: 10.1097/FJC.0b013e31820d1b89 